\title{
Hypoxemia associated with lumbar puncture in neonates
}

\author{
Tripti Sharma1, Mohit Ajmera², Gopikishan Sharma², Amrita Mayenger ${ }^{3}$ \\ From ${ }^{1}$ Junior Resident, ${ }^{2}$ Assistant Professor, ${ }^{3}$ Professor and Head, Department of Pediatrics, Government Medical College, Kota, Rajasthan, India
}

\begin{abstract}
Objectives: The objectives of the study were to study the effect of different stages of lumber puncture (LP) procedure on oxygen saturation $\left(\mathrm{SpO}_{2}\right)$ in newborn baby. Materials and Methods: It was a 1-year, prospective observational study conducted in a tertiary care hospital. Forty neonates were included who fulfilled the inclusion criteria. $\mathrm{SpO}_{2}$ was measured during the different stages of LP in the recruited newborns and comparison was done between $\mathrm{SpO}_{2}$ changes in LP duration of $<5$ min and more than 5 min groups and the effect on $\mathrm{SpO}_{2}$ in preterm and term babies during LP. Results: Mean $\mathrm{SpO}_{2}$ during baseline, flexion, needle insertion, immediate repositioning, and $5 \mathrm{~min}$ after procedure were 94.5, 91.82, 88.92, 94.7, and 95.38, respectively. When compare to baseline, drop in $\mathrm{SpO}_{2}$

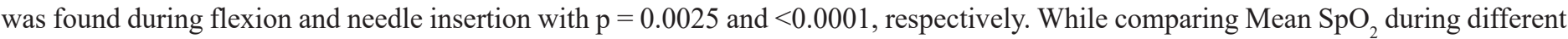
phases between duration of $\mathrm{LP}>5$ min versus $<5$ min group, the saturation was less during flexion positioning with maximum fall at the time of needle insertion in LP duration $>5$ min. The regain of saturation during repositioning was more in LP $<5$ min versus duration $>5$ min group. Comparing between LP duration $>5$ min versus $<5$ min group, fall in $\mathrm{SpO}_{2}$ from baseline ( 5 min before LP procedure) was more in LP duration $>5$ min group during flexion and needle insertion phase. Hypoxemia is more in preterm than term neonates during flexion and during needle insertion, supine repositioning. Furthermore, hypoxia recovered after 5 min of supine repositioning more in term infants. Conclusions: Significant hypoxia was associated in newborn in flexion and needle insertion phase of lumbar puncture, especially in preterm newborns and those with prolonged duration of procedure.
\end{abstract}

Key words: Hypoxia, Lumbar puncture, Meningitis, Newborn

S epticemia is the third leading cause of mortality in the newborn in India, next only to prematurity and birth asphyxia. In the developing countries, the incidence is higher, at $0.8-6.1$ per 1000 live births, with a mortality rate of up to $58 \%$ [1]. Mortality can be as high as $40 \%$ in meningitis cases which has declined over the last decades. Morbidity rate is around $20-60 \%$ including cerebral palsy and neurodevelopment impairment. Clinical features of septicemia and meningitis often overlap. Meningitis is more common in neonates evaluated for late-onset sepsis compared to those presenting with early-onset sepsis. However, blood cultures are negative in 10-20\% of cases of neonatal meningitis and selective evaluation of culture-proven bacteremia can result in missed diagnosis. Cerebrospinal fluid (CSF) culture obtained by lumbar puncture (LP) is the gold standard for diagnosis of meningitis as well as for the identification of pathogens and appropriate choice of therapy [2]. Hence, it is important to include LP for any newborn with signs of CNS infection and late-onset septicemia.

Examination of the CSF and measurement of the pressure, it creates in the subarachnoid space, are essential in confirming the diagnosis

\section{Access this article online}

Received - 06 December 2021

Initial Review - 14 December 2021

Accepted - 18 December 2021

DOI: $10.32677 / \mathrm{ijch} . v 8 \mathrm{i} 12.3214$ of meningitis, encephalitis, and idiopathic intracranial hypertension and it is often helpful in assessing subarachnoid hemorrhage. Having an experienced assistant who can position, restrain, and comfort, the patient is critical to the success of the procedure.

While LP has many advantages, it also has many risks associated with the procedure, especially in a newborn. It's always been a matter of concern - Is LP feasible in a critically ill newborn? Certain risks are associated with LP and these risks increase with slight manipulations or changes in the position of the newborn, increased duration of LP, and decreasing maturity of the neonate. Of the risk associated with LP, the highest concern is that of hypoxemia during LP could be the result of flexion position or the invasive nature of the procedure and the associated pain production [3]. Our study aims at determining the effect of LP procedure, LP duration, and maturity of newborn on oxygen saturation changes.

\section{MATERIALS AND METHODS}

This prospective observational study was conducted in Neonatal Intensive Care Unit (NICU) of Jay Kay Lon Hospital, Kota, from

Correspondence to: Dr. Mohit Ajmera, Department of Pediatrics, 4-C-37 Mahaveer Nagar Extension, Kota, Rajasthan - 324 009, India. E-mail: ajmeramohit88@gmail.com

(C) 2021 Creative Commons Attribution-NonCommercial 4.0 International License (CC BY-NC-ND 4.0). 
March 1, 2019, to February 28, 2020. All neonates admitted into the NICU of our institution with suspected late-onset sepsis with positive sepsis screen and/or meningitis symptoms were included and newborns with respiratory and cardiovascular compromise, local skin site infection, presence of lumbar malformation, recurrent apnea, bradycardia, and negative consent were excluded from the study. The Institutional Ethics Committee approved the study. After getting written informed consent from the parents, neonates were recruited for the study.

Hypoxemia was defined as oxygen desaturation $\left(\mathrm{SpO}_{2}\right)$ in the blood which is characterized by pulse oximeter reading $\left(\mathrm{SpO}_{2}\right.$ of $<95 \%)$.

\section{Sepsis}

Late-onset sepsis was suspected on the basis of the clinical signs and symptoms such as presence of hypothermia (or fever), hypoglycemia, feeding intolerance, altered sensorium, convulsion, and fast breathing with onset of symptoms after 3 days of life. Each suspected sepsis baby underwent sepsis screen and enrolled only after sepsis screen was positive [4].

\section{Sepsis Screen}

(1) Total leukocyte count $<5000 / \mathrm{mm}^{3}$, (2) low absolute neutrophilic count as per Manroe chart for term and Mouzinho's chart for VLBW infants, (3) immature to total neutrophil ratio $>0.2$, (4) micro-ESR $>15 \mathrm{~mm}$ in the $1^{\text {st }} \mathrm{h}$, and (5) C-reactive protein $>1 \mathrm{mg} / \mathrm{dl}$. Positive sepsis screen was considered when any two parameters were abnormal [4].

Patients were assessed for hypoxemia and clinical deterioration by measuring cardiorespiratory changes (heart rate and respiratory rate) and $\mathrm{SpO}_{2}$ using pulse oximeter (Nellcor technology) on the right upper hand by wrap around sensor probe. Relevant clinical information was recorded including age, sex, anthropometry, relevant maternal, perinatal and neonatal history suggestive of risk factors for sepsis, and examination findings at presentation recorded in the predesigned data sheet.

Antenatal history of infections including TORCH, drug intake, pregnancy-induced hypertension (PIH), gestational diabetes, and intrauterine growth status was taken. Peripartum PIH, antepartum hemorrhage, chorioamnionitis, and fetal distress (fetal heart rate abnormalities or meconium-stained liquor) were recorded. Mode of presentation and delivery noted. Resuscitation details if done also recorded. Examination findings were noted. Management of neonates was done as per unit protocol.

LP was conducted under sterile conditions in the NICU. Neonates enrolled in the study were placed on the table with sterile cloth with pulse oximeter (Covidien Nellcor) attached to the right upper hand with wrap around sensor before the procedure, and the $\mathrm{SpO}_{2}$ was noted. Neonates were positioned in the lateral recumbent position drawing the knees up to the chest. For procedural analgesia, dextrose was given orally 2 min before the start of the procedure, with repeated doses if required. Readings of the $\mathrm{SpO}_{2}$ as shown by the pulse oximeter were recorded $5 \mathrm{~min}$ before the procedure (baseline); during flexion, during needle insertion; during repositioning into supine position after CSF collection; and 5 min after been in supine position and also total duration of procedure noted from attainment of flexion position to 5 min after supine reposition as LP procedure duration. Standard protocol was followed: The readings were obtained from using appropriate size probe attached to the right upper hand with wrap around sensor probe.

\section{Statistical Analysis}

Statistical analysis was performed using SPSS Window version 21. Association of different aspects was calculated by Student's t-tests and Mann-Whitney U-test for quantitative data and Chi-square test for qualitative data. $\mathrm{p}<0.05$ was considered statistically significant.

\section{RESULTS}

A total of 60 neonates were screened of which 40 neonates were enrolled while 20 cases were excluded (13 cardiopulmonary compromised state, two local sites infection, and five neonates' parental consent were not given). Twenty-five babies out of $40(62.5 \%)$ were preterm; out of which, seven were $28-32$ weeks preterm, while 18 were $32-37$ weeks preterm. Term babies were comprised $37.5 \%$. In our study, 17 (42.5\%) were male and $23(57.5 \%)$ females with male-to-female ratio of 1:1.35. Mean weight of the neonates was $2.477 \pm 0.53 \mathrm{~kg}$. Mean length of the neonates was $34.01 \pm 5 \mathrm{~cm}$ and mean head circumference was $45.42 \pm 4.57 \mathrm{~cm}$. Among 40 neonates, $23(57.5 \%)$ were appropriate for gestational age and $17(42.5 \%)$ were small for gestational age (SGA). Out of 17 SGA babies, 15 (88.23\%) were asymmetrical IUGR and $2(11.76 \%)$ were symmetrical IUGR babies.

Among the associated clinical features of septic babies undergoing LP, lethargy is the most common symptom (72\%) followed by jaundice (32\%) and convulsions (21\%), fever (15\%), and hypothermia (11\% babies). Mean $\mathrm{SpO}_{2} 5 \mathrm{~min}$ before positioning for LP, during flexion, during insertion, and during reposition were $94.5 \pm 4.51,91.82 \pm 4.14,88.92 \pm 4.34$, and $94.7 \pm 3.23$, respectively, and recovered to mean of $95.375 \pm 3.014$ at 5 min after LP. Comparing the $\mathrm{SpO}_{2}$ during various phases of LP from baseline ( 5 min before LP) showed that the extent of desaturation was greatest during the stage of needle insertion as per Table 1.

It was observed that desaturation was more commonly seen in preterm babies in comparison to the term babies. During the procedure, hypoxemia occurred in $32 \%$ of preterm babies as compared to $26.6 \%$ of term babies. Comparing the level of desaturation between term and preterm babies at different steps of the LP also yield significant results with preterm undergoing more desaturation as compared to the term babies as per Table 2 .

The data in Table 3 illustrate about comparison of mean saturation values during different phases of LP with the duration 
Table 1: Comparing the mean values of $\mathrm{SpO}_{2}$ of during the various phases of LP

\begin{tabular}{lcccc}
\hline Stages & $\begin{array}{c}\text { Mean } \\
\mathbf{S p O}_{2}\end{array}$ & SD & $\begin{array}{c}\text { Mean } \\
\text { difference }\end{array}$ & p-value $^{\#}$ \\
\hline During flexion versus & 91.82 & 4.14 & 2.73 & $0.0025^{*}$ \\
5 min before LP & 94.55 & 4.51 & & \\
During insertion versus & 88.92 & 4.34 & 5.625 & $<0.0001^{*}$ \\
5 min before LP & 94.55 & 4.51 & & \\
During reposition versus & 94.7 & 3.23 & -0.15 & 0.864 \\
5 min before LP & 94.55 & 4.51 & & \\
5 min after LP versus & 95.375 & 3.01 & -0.825 & 0.339 \\
5 min before LP & 94.55 & 4.51 & & \\
\hline
\end{tabular}

\#Student's t-test, * $\mathbf{P}<0.05$

Table 2: Effect of maturity of oxygen saturation changes during LP changes

\begin{tabular}{lccc}
\hline Phases of LP & \multicolumn{2}{c}{ Mean SpO } & \multirow{2}{*}{ p-value $^{\#}$} \\
\cline { 2 - 3 } & Preterm (25) & Term (15) & \\
\hline 5 min before LP & $94.8 \pm 4.35$ & $96.8 \pm 3.23$ & 0.1316 \\
During flexion & $90.2 \pm 3.76$ & $94.6 \pm 4.59$ & $0.0021^{*}$ \\
During insertion & $87.8 \pm 3.49$ & $92.4 \pm 5.53$ & $0.0025^{*}$ \\
During reposition to supine & $92.6 \pm 3.33$ & $95 \pm 3.27$ & $0.0324^{*}$ \\
5 min after LP & $94 \pm 2.61$ & $96.6 \pm 2.47$ & $0.0035^{*}$ \\
\hline
\end{tabular}

\#Student's t-test, *p<0.05

Table 3: Comparing the mean $\mathrm{SpO}_{2}$ values during the various phases of $L P$ in $L P$ duration $>5$ min versus $<5$ min groups

\begin{tabular}{lccccc}
\hline $\begin{array}{l}\text { Phase of } \\
\text { LP }\end{array}$ & $\begin{array}{c}\text { LP } \\
\text { duration (min) }\end{array}$ & Min & Max & Mean \pm SD & p-value \\
\hline 5 min & $>5$ & 88 & 100 & $94.57 \pm 3.33$ & 0.43 \\
before LP & $<5$ & 86 & 99 & $95.14 \pm 4.13$ & \\
During & $>5$ & 84 & 98 & $92.62 \pm 3.71$ & 0.152 \\
flexion & $<5$ & 84 & 99 & $94.14 \pm 4.26$ & \\
During & $>5$ & 80 & 94 & $88.92 \pm 3.72$ & $0.0121^{*}$ \\
insertion & $<5$ & 82 & 99 & $92.36 \pm 5.05$ & \\
During & $>5$ & 85 & 99 & $92.42 \pm 3.41$ & $0.0010^{*}$ \\
reposition & $<5$ & 92 & 99 & $96.5 \pm 2.9$ & \\
5 min & $>5$ & 90 & 99 & $95.77 \pm 2.55$ & 0.412 \\
after LP & $<5$ & 92 & 99 & $96.43 \pm 2.41$ & \\
\hline
\end{tabular}

\#Mann-Whitney U-test *p<0.05

of LP (from the start of the attainment of the flexion position to $5 \mathrm{~min}$ after the repositioning of the neonate into the supine position). The saturation gradually starts to fall during flexion positioning with maximum fall at the time of needle insertion in LP duration $>5$ min. Furthermore, it was observed that the regain of saturation during repositioning was more in LP lasting $<5 \mathrm{~min}$ (Table 3).

The duration of LP also affected the levels of desaturation from baseline during different phases of LP. Fall in oxygen saturation from baseline ( $5 \mathrm{~min}$ before LP procedure) was significantly more in LP duration $>5$ min group as compared to LP $<5$ min group during flexion and needle insertion phase (Table 4).
Table 4: Comparing the duration of lumbar puncture and difference in mean values of oxygen saturation from baseline during the various phases of LP

\begin{tabular}{lccc}
\hline $\begin{array}{l}\text { Phase of } \\
\text { LP }\end{array}$ & $\begin{array}{c}\text { LP } \\
\text { duration }\end{array}$ & $\begin{array}{c}\text { Mean difference in SpO } \\
\pm \text { SD (from baseline, i.e., } \\
\text { 5 min before LP) }\end{array}$ & p-value $^{\#}$ \\
\hline During & $>5$ & $1.96 \pm 1.04$ & $0.0036^{*}$ \\
flexion & $<5$ & $1 \pm 0.68$ & \\
During & $>5$ & $5.65 \pm 2.019$ & $0.0001^{*}$ \\
insertion & $<5$ & $2.79 \pm 1.81$ & \\
During & $>5$ & $2.15 \pm 2.19$ & 0.3665 \\
reposition & $<5$ & $1.57 \pm 1.28$ & \\
5 min after & $>5$ & $1.81 \pm 1.39$ & 0.5311 \\
procedure & $<5$ & $2.14 \pm 1.88$ & \\
\hline
\end{tabular}

\#Student's t-test, *p $<0.05$

\section{DISCUSSION}

Septicemia is the third leading cause of neonatal mortality in India. Signs and symptoms of meningitis and septicemia can overlap so screening for meningitis in all cases of late-onset sepsis is recommended [4]. In our study, preterm (62.5\%) had more sepsis as compared to term (37.5\%). Preterm newborns were 3.36 times more likely to develop neonatal sepsis compared to term newborns. The possible explanation is that preterm babies have immature immune systems (low neutrophil storages) and body organs that fight infections [5]. The most common symptom in septic babies undergoing LP was lethargy (72\%) followed by jaundice (32\%) and convulsions (21\%). These were similar to the study conducted by Shaw et al. whereas a history of decreased activity and feeding problems was the most common predictors followed by fever and respiratory signs [6].

While assessing for $\mathrm{SpO}_{2}$ changes during various phases of LP, significant hypoxemia was associated with LP; especially, during flexion and needle insertion phase which is similar to the study conducted by Duru et al. [7]. Before positioning for LP, mean $\mathrm{SpO}_{2}$ was as $94.5 \pm 4.51$. After positioning, $\mathrm{SpO}_{2}$ reduced to $91.82 \pm 4.14$ which further decreased to $89.92 \pm 4.34$ at the time of insertion of spinal needle and again improved to $94.7 \pm 3.23$ after supine repositioning. Bedettiet et al. also found with similar results about desaturation during LP [8].

Comparing the $\mathrm{SpO}_{2}$ changes during various phases of LP from baseline ( $5 \mathrm{~min}$ before LP) showed that the extent of desaturation was greatest during the stage of needle insertion $(p<0.0001)$ followed by during flexion $(\mathrm{p}=0.0025)$. Similar findings were observed by Duru et al. [7] with the highest desaturation observed with needle insertion during LP.

It was observed that desaturation was more commonly seen in preterm babies in comparison to the term babies. About $32 \%$ of preterm babies desaturated as compared to $26.6 \%$ term indicating the higher level of susceptibility to hypoxemia in preterm babies while undergoing the procedure. These are similar to the findings by Bedettiet et al. [8] reporting more desaturation in lower gestational age babies. 
Desaturation during LP in neonates can be attributed to physiological challenges such as small functional residual capacity, higher metabolic demand, unfavorable rib configuration, inefficient respiratory muscles, especially intercostal muscles, smaller and highly compliant airway, and immature control of respiration and periodic breathing which are more pronounced in preterm neonates. Inspiration occurs almost entirely because of diaphragmatic descent which is greatly restricted because of splinting of diaphragm in flexion position in LP [7,9]. Physiology of greater fall in $\mathrm{SpO}_{2}$ during needle insertion phase is not clearly understood but increased oxygen consumption and demand and ventilation perfusion mismatch because of pain during needle insertion can be attributing factors $[7,10]$.

When comparing mean $\mathrm{SpO}_{2}$ in different phases of LP in between two groups of total LP duration of $<5$ min versus $>5 \mathrm{~min}$, it was found that hypoxia was significantly more in $>5$ min duration group while resolution of hypoxia was significantly better on immediate supine repositioning after procedure in $<5$ min LP duration group $(\mathrm{p}=0.001)$. Duru et al. [7] also concluded with maximum desaturation observed at the point of needle insertion in $>5$ min $\mathrm{LP}$ while $\mathrm{SpO}_{2}$ was lower in those with prolonged procedure after attainment of supine position. Reason behind this observation can be attributed to the duration of procedure and longer respiratory compromised state and higher consumption of respiratory reserve due to prolong duration of procedure.

We also found that the level of desaturation from baseline ( 5 min before LP) was significantly more in LP $>5$ min group as compared to $<5$ min group during flexion and needle insertion phase of LP ( $p=0.0036$ and 0.0001 , respectively). There was no significant difference in oxygen saturation was found between the two groups during Immediate reposition and $5 \mathrm{~min}$ after procedure. Duru et al. [7] in their study also had similar findings with significant desaturation observed during needle insertion and regain of saturation with repositioning in $>5$ min duration group.

The strength of our study was that it was done on hemodynamic stable babies so effects on oxygen saturation can reliably be attributed to LP. This study also included comparison between term and preterm neonates. It also has certain limitations as the study was done on a small number of patients and for limited duration. The study can be extended with larger sample size to validate the result of the present study.

\section{CONCLUSIONS}

Lumbar puncture in neonates is associated with significant hypoxia; especially, during the flexion and needle insertion phase. Preterm neonates are significantly more prone to hypoxia during flexion and needle insertion phase of the procedure and resolution of hypoxia during immediate and $5 \mathrm{~min}$ after repositioning to supine is lesser than in the term neonates. Duration of LP of $>5 \mathrm{~min}$ was significantly associated with more hypoxia in needle insertion phase and lesser improvement after immediate repositioning to supine after procedure as compared to $<5$ min duration. Fall of $\mathrm{SpO}_{2}$ was also significantly more in $\mathrm{LP}$ of $>5$ min duration group during flexion and needle insertion from baseline.

\section{REFERENCES}

1. Ku LC, Boggess KA, Cohen-Wolkowiez M. Bacterial meningitis in infants. Clin Perinatol 2015;42:29-45.

2. Doherty CM, Forbes RB. Diagnostic lumbar puncture. Ulster Med J 2014;83:93-102.

3. Marshall AS, Sadarangani M, Scrivens A, Williams R, Yong J, Bowler U, et al. Study protocol: NeoCLEAR: Neonatal champagne lumbar punctures every time-an RCT: A multicentre, randomised controlled $2 \times 2$ factorial trial to investigate techniques to increase lumbar puncture success. BMC Pediatr 2020;20:165.

4. Sankar MJ, Agarwal R, Deorari AK, Paul VK. Sepsis in the newborn. Indian J Pediatr 2008;75:261-6.

5. Belachew A, Tewabe T. Neonatal sepsis and its association with birth weight and gestational age among admitted neonates in Ethiopia: Systematic review and meta-analysis. BMC Pediatr 2020;20:55.

6. Shaw CK, Shaw P, Malla T, Malla KK. The clinical spectrum and outcome of neonatal sepsis in a neonatal intensive care unit at a tertiary care hospital in western Nepal: January 2000 to December 2005-a retrospective study. East J Med 2012;17:119-25.

7. Duru CO, Ederiane OE, Allen-Ameri D, Ibrahim ZF, Pondei K, Aliyu I. Does hypoxemia occur before, during or after lumbar puncture procedure in ill newborns? Our experience in a tertiary health center. J Clin Neonatol 2017;6:173-8.

8. Bedetti L, Lugli L, Marrozzini L, Baraldi A, Leone F, Baroni L, et al. Safety and success of lumbar puncture in young infants: A prospective observational study. Front Pediatr 2021;9:692652.

9. Saikia D, Mahanta B. Cardiovascular and respiratory physiology in children. Indian J Anaesth 2019;63:690-7.

10. Mathew PJ, Mathew JL. Assessment and management of pain in infants. Postgrad Med J 2003;79:438-43.

Funding: None; Conflicts of Interest: None Stated.

How to cite this article: Sharma T, Ajmera M, Sharma G, Mayenger A. Hypoxemia associated with lumbar puncture in neonates. Indian $\mathrm{J}$ Child Health. 2021; 8(12):408-411. 